\title{
GENERALIZATIONS OF TWO THEOREMS OF JANISZEWSKI
}

\author{
R. H. BING
}

Janiszewski proved [2] ${ }^{1}$ the following for the plane.

THEOREM A. The sum of two compact continua does not cut the point $A$ from the point $B$ provided neither cuts $A$ fram $B$ and their common part is connected or does not exist.

TheOREM B. The sum of two compact continua cuts the plane provided their common part is not connected.

Many generalizations and modifications of these theorems appear in the literature (for example, see $[1,3,4,6,8,9,10,11,12]$ ). This paper generalizes the two theorems by considering, instead of continua, sets that are neither open nor closed. All sets referred to in this paper are in the plane.

Definition. The set $R$ cuts the point $A$ from the point $B$ in the set $W$ provided some continuum in $W$ contains $A$ and $B$ and $R$ intersects neither $A$ nor $B$ but it intersects every continuum in $W$ containing $A$ and $B$. If $R$ cuts $A$ from $B$ in the plane, we simply say that it cuts $A$ from $B$. Thus we use $c u t$ in the sense that coupe was used in Fundamenta Mathematicae (for example, see [1, p. 75, and 10, p. 15]) and not in the sense used by some writers to mean separate. If a closed set cuts two points from each other in the plane, it separates them from each other; this is not true for more general sets.

Generalizations of Theorem A. We shall make use of the following known result [12, p. 129, and 4, pp. 35-36].

TheOREM 1. If neither of the closed point sets $H$ and $K$ separates the point $A$ from the point $B$, the common part of $H$ and $K$ is connected or does not exist and the part of $H$ in the complement of $K$ is compact, then the sum of $H$ and $K$ does not separate $A$ from $B$.

TheOREM 2. If neither of the point sets $H$ and $K$ separates the point $A$ from the point $B$, each of the sets is closed in their sum, ${ }^{2}$ one of the sets is compact ${ }^{3}$ and their common part is a continuum or does not exist, then the sum of $H$ and $K$ does not separate $A$ from $B$.

\footnotetext{
Presented to the Society, September 17, 1945; received by the editors July 24, 1945.

${ }^{1}$ Numbers in brackets refer to the references cited at the end of the paper.

${ }^{2}$ The set $H$ is closed in the set $S$ if $\bar{H} \cdot S$ is equal to $H$.

${ }^{3}$ In Theorems 2, 5, 6, 7, 8, 9, 10 instead of assuming that one of the sets is compact, we can assume that the part of it in the complement of the other is compact.
} 
Proof. Assume that $H+K$ separates $A$ from $B$. Then $H+K$ contains a continuum $M$ separating $A$ from $B[8$, Theorem 73, p. 150, and Theorem 24, p. 194]. However, neither of the closed point sets $M \cdot H+H \cdot K$ and $M \cdot K+H \cdot K$ separates $A$ from $B$ because neither $H$ nor $K$ does. Hence, by Theorem 1 their sum does not. But their sum contains $M$.

Example. Theorem 2 would not be true if instead of requiring that $H \cdot K$ be a continuum we only required that it be connected. Let $H \cdot K$ be the sum of the interval from $(0,0)$ to $(-1,2)$, the interval from $(-1,2)$ to $(1,1)$ and the image of

$$
y=\sin (\pi / 2 x), \quad 0<x \leqq 1 ;
$$

let $H-H \cdot K$ be the image of

$$
0 \leqq y<\sin (\pi / 2 x), \quad 0<x \leqq 1 ;
$$

let $K-H \cdot K$ be the image of

$$
0 \geqq y>\sin (\pi / 2 x), \quad 0<x \leqq 1 .
$$

THEOREM 3. If the sum of the simple closed curve $J$ and the arc $P Q$ separates the point $A$ from the point $B$ then their sum contains a simple closed curve $J^{\prime}$ such that $J^{\prime}$ minus the common part of $J^{\prime}$ and $P Q$ is a connected subset of $J$.

Proof. There is a subset $M$ of $J$ irreducible with respect to $M+P Q$ separating $A$ from $B$. Also, $M+P Q$ contains a continuum $J^{\prime}$ which is irreducible with respect to separating $A$ from $B$. This continuum is a simple closed curve containing $M$. If $M$ were the sum of two mutually separated point sets $M_{1}$ and $M_{2}$, neither of the closed sets $M_{1}+P Q$ and $M_{2}+P Q$ would separate $A$ from $B$ and their sum would not. As $M$ is irreducible with respect to $M+P Q$ separating $A$ from $B$, it contains no point of $P Q$ and is therefore equal to $J^{\prime}-J^{\prime} \cdot P Q$.

ThEOREM 4. If the points $A$ and $B$ belong to the same component of the complement of the connected domain $D$ and $J$ is a simple closed curve separating $A$ from $B$ then $J$ contains a continuum which contains no point of $D$ and which cuts $A$ from $B$ in the complement of $D .4$

Proof. Assume that $J$ intersects $D$; the theorem is obviously true if it does not. Let $O_{1}, O_{2}, \cdots$ be a sequence of points dense in $D \cdot J$. There is an arc $O_{1} O_{2}$ in $D$ and by Theorem 3 there is a simple closed curve $J^{\prime}$ separating $A$ from $B$ and such that $J^{\prime}-J^{\prime} \cdot O_{1} O_{2}$ is an open

\footnotetext{
${ }^{4}$ By Theorem 7 it follows that $J$ contains a continuum cutting $A$ from $B$ in the complement of $D$ and intersecting $\bar{D}$ in no more than two points.
} 
arc $\left(P_{1} Q_{1}\right)$ of $J$ containing neither $O_{1}$ nor $O_{2}$. If $P_{1}$ is not $O_{3}$ there is an arc $P_{1} O_{3}$ in $D$ and there is a simple closed curve $J^{\prime \prime}$ separating $A$ from $B$ such that $J^{\prime \prime}-J^{\prime \prime} \cdot\left(O_{1} O_{2}+P_{1} O_{3}\right)$ is an open arc $\left(P_{2} Q_{2}\right)$ of $\left(P_{1} Q_{1}\right)$. This process is continued to get an infinitude of $\left(P_{n} Q_{n}\right)$ 's where $\left(P_{n} Q_{n}\right)$ is a subset of $\left(P_{n-1} Q_{n-1}\right)$ containing neither $O_{1}, O_{2}, \cdots$, nor $O_{n+1}$ but separating $A$ from $B$ in the complement of $D$.

The common part $M$ of $\left(P_{1} Q_{1}\right),\left(P_{2} Q_{2}\right), \cdots$ is the same as the common part of $P_{1} Q_{1}, P_{2} Q_{2}, \cdots$ and is therefore a continuum. As no point of $D$ belongs to each $P_{n} Q_{n}, M$ does not intersect $D$. If $C$ is a continuum containing $A$ and $B$ but no point of $D$, then for each positive integer $n$ it contains a point of $P_{n} Q_{n}$ and therefore contains a point of $M$.

Example. Theorem 4 would not be true if the word "cuts" were replaced by "separates." Consider the domain $D$ which is the image of

$$
\sin (\pi / x)<y<1+\sin (\pi / x), \quad 0<x<1,
$$

and a circle $J$ with center at $(0,0)$ and radius 1 . Now $J$ contains no continuum which does not intersect $D$ but which separates $(0,0)$ from $(2,0)$ in the complement of $D$.

TheOREM 5. The sum of two domains does not separate the point $A$ from the point $B$ provided neither of the domains separates $A$ from $B$, one of them is compact and their common part is connected or does not exist.

Proof. First, assume that $H$ and $K$ are two compact domains satisfying the above hypotheses whose sum separates $A$ from $B$. Then $H+K$ contains a simple closed curve $[8$, Theorem 9, p. 184] separating $A$ from $B$. By Theorem 4 , this simple closed curve contains a continuum $M$ which contains no point of $H \cdot K$ but which cuts $A$ from $B$ in the complement of $H \cdot K$. As $M$ contains no point of $H \cdot K$, it is a subset of either $H$ or $K$, say $H$. Then the domain $H$ cuts $A$ from $B$ and therefore separates $A$ from $B$.

The theorem may be proved in the case in which only one of $H, K$ is compact by regarding the plane as a sphere minus one point.

Theorem 6. Suppose that $H$ and $K$ are sets such that one is compact, neither cuts the point $A$ from the point $B$, their common part is connected and on the subtraction of their common part from each, the remainders are mutually separated. Then the sum of $H$ and $K$ does not cut $A$ from $B .^{5}$

\footnotetext{
- Using other methods, Eilenberg showed [1, Theorem 8, p. 78] that the sum of the two compact sets $H$ and $K$ does not cut $A$ from $B$ provided neither cuts $A$ from $B$, each is open or closed in $H+K$ and $H \cdot K$ is connected.
} 
Proof. Suppose that $C_{H}$ and $C_{K}$ are continua such that each contains $A+B, C_{H}$ does not intersect $H$ and $C_{K}$ does not intersect $K$. There exist domains $D_{0}, D_{1}, D_{2}$ such that $D_{0}$ is connected and covers $H \cdot K$ but no point of $C_{H}+C_{K}, D_{1}$ covers $H$ but no point of $C_{H}, D_{2}$ covers $K$ but no point of $C_{K}, D_{1}-D_{0} \cdot D_{1}$ and $D_{2}-D_{0} \cdot D_{2}$ are mutually separated and one of $D_{1}, D_{2}$ is compact. Considering $D_{0}+D_{1}$ and $D_{0}+D_{2}$ as the domains of Theorem 5 , we find that $D_{0}+D_{1}+D_{2}$ does not cut $A$ from $B$. Neither does $H+K$ which is a subset of $D_{0}+D_{1}$ $+D_{2}$.

THEOREM 7. If $H$ is a compact closed set cutting the point $A$ from the point $B$ in the complement of the connected set $K$, then $H$ contains a subset $H^{\prime}$ irreducible with respect to being a closed set cutting $A$ from $B$ in the complement of $K$. Also, $H^{\prime}$ is a continuum that is not separated by any subset of the closure of $K$.

PRoof. Let $D_{1}, D_{2}, \cdots$ be a sequence of domains such that if $D$ is a domain containing a point $P$ of $H$, there exists an integer $i$ such that $D_{i}$ is a subset of $D$ containing $P$. Let $H_{1}$ be a closed subset of $H$ such that (1) $H_{1}$ cuts $A$ from $B$ in the complement of $K$ and (2) $H_{1}$ intersects $D_{1}$ only if every closed subset of $H$ satisfying (1) intersects $D_{1}$. In general, let $H_{n}$ be a closed subset of $H_{n-1}$ such that (1) $H_{n}$ cuts $A$ from $B$ in the complement of $K$ and (2) $H$ intersects $D_{n}$ only if every closed subset of $H_{n-1}$ satisfying (1) intersects $D_{n}$.

The common part $H^{\prime}$ of $H_{1}, H_{2}, \cdots$ is a closed subset of $H$. It cuts $A$ from $B$ in the complement of $K$ for each continuum in the complement of $K$ containing $A$ and $B$ intersects each of $H_{1}, H_{2}, \cdots$. If $H^{\prime}$ contained a proper closed subset $M$ cutting $A$ from $B$ in the complement of $K$, then there would be an integer $i$ such that $D_{i}$ contained a point $P$ of $H^{\prime}$ but no point of $M$. Then $H_{i}$ would not contain $P$.

Assume that $\bar{K}$ contains a subset $R$ such that $H^{\prime}-R$ is the sum of two mutually separated point sets $E_{1}$ and $E_{2}$. Then there exist mutually exclusive domains $F_{1}$ and $F_{2}$ containing $E_{1}$ and $E_{2}$ respectively. However, as neither $K+\left(H^{\prime}-H^{\prime} \cdot F_{1}\right)$ nor $K+\left(H^{\prime}-H^{\prime} \cdot F_{2}\right)$ cuts $A$ from $B$, then by Theorem $6, K+H^{\prime}$ could not.

Generalizations of Theorem B. We shall now consider some generalizations of Theorem B.

Definition. The junction [5, p. 206] of the sets $H$ and $K$ is the common part of $H$ and the closure of $K$ plus the common part of $K$ and the closure of $H$, that is $H \cdot \bar{K}+\bar{H} \cdot K$.

THEOREM 8. If $H$ and $K$ are connected sets one of which is compact 
and such that $H$ is continuumwise connected and the junction of $H$ and $K$ is the sum of two mutually separated sets each of which contains a point of $H$, then the sum of $H$ and $K$ cuts the plane. ${ }^{6}$

Proof. Let the junction $M$ of $H$ and $K$ be the sum of two mutually separated point sets $M_{A}$ and $M_{B}$ which contain points $A$ and $B$ respectively of $H$. There exists [7, Corollary 2, p. 221] a compact continuum $J$ irreducible with respect to separating $A$ from $B$ and such that if the components of $\bar{M}_{A} \cdot \bar{M}_{B}$ are regarded as points, $J$ is a simple closed curve (or a point) containing no point of $\bar{M}-\bar{M}_{A} \cdot \bar{M}_{B} \cdot$. Let $T$ be the set of all continua $t$ such that $t$ is either a point of $J$ not belonging to $\bar{M}$ or a component of $\bar{M}_{A} \cdot \bar{M}_{B}$.

Let $C$ be a continuum in $H$ containing $A$ and $B$. No point of $C \cdot J$ is a limit point of $K$, for $J$ contains no point of $M$. Using an argument similar to that employed by Kuratowski [7, Theorem 11, pp. 222223 ] we find that $T$ contains a finite number of elements $t_{1}, t_{2}, \cdots, t_{n}$ none of which intersect $C+K$ and each of which belongs both to the boundary of a component of $J-\left(t_{1}+\cdots+t_{n}\right)$ which contains a point of $C$ but no point of $K$ and also to the boundary of a component of $J-\left(t_{1}+\cdots+t_{n}\right)$ which contains a point of $K$ but no point of $C$. Let $W_{C}$ be the sum of those components containing a point of $C$ and let $W_{K}$ be the sum of those containing a point of $K$.

Assume that $C+K$ does not cut between any two elements of $t_{1}, t_{2}, \cdots, t_{n}$. Then there is a continuum $V$ containing $t_{1}+t_{2}+\cdots$ $+t_{n}$ but no point of $C+K$. As neither the continuum $V+W_{C}$ nor the continuum $V+W_{H}$ separates $A$ from $B$, then by Theorem 1 their sum does not. However, their sum contains $J$.

Let $i$ and $j$ be integers such that $C+K$ cuts $t_{i}$ from $t_{j}$ and let $\alpha_{i}$ and $\alpha_{j}$ be maximal connected subsets of $J$ containing $t_{i}$ and $t_{j}$ respectively but no point belonging to an element of $T$ containing a point of $C+K$. Now $C+K$ cuts every point of $\alpha_{i}$ from every point of $\alpha_{j}$. But $\alpha_{m}(m=i, j)$ contains a point $P_{m}$ not belonging to $H$ for $J$ contains no point of $M$. Therefore $H+K$ cuts $P_{i}$ from $P_{j}$.

Example. Theorem 8 would not be true if we did not require that $H$ be continuumwise connected. Let $H$ be the sum of the intervals having end points at $(-3,0)$ and $(-3,1)$, at $(-3,0)$ and $(-1,0)$, at $(1,0)$ and $(2,0)$ and $\left(-3,1 / 2^{n}\right)$ and $\left(2,1 / 2^{n}\right)$ where $n=1,2, \cdots$. Let $K$ be the sum of the intervals having end points at $(3,0)$ and $(3,1)$, at $(1,0)$ and $(3,0)$, at $(-2,0)$ and $(-1,0)$ and at $\left(-2,1 / 3^{n}\right)$ and $\left(3,1 / 3^{n}\right)$.

${ }^{6}$ Nicodym proved this theorem in the case where $H$ is a bounded continuum [10, Theorem 2, p. 20]. 
THEOREM 9. If $H$ and $K$ are connected sets one of which is compact, the common part of the closure of $H$ and the closure of $K$ is the sum of two mutually separated point sets each of which contains a point of the junction of $H$ and $K$, then $H+K$ cuts the plane.

PRoof. There exists a simple closed curve containing no point of $\bar{H} \cdot \bar{K}$ and separating some two points of the junction of $H$ and $K$. Using an argument similar to that used in Theorem 8 we find that this simple closed curve contains two points which $H+K$ cuts from each other.

THEOREM 10. The sum of two continuumwise connected sets separates the plane provided one of them is compact and their common part exists but does not belong to any continuumwise connected subset of their junction.

Proof. Suppose that $H$ and $K$ are sets satisfying the hypotheses of Theorem 10 and that the complement of $H+K$ is connected. Neither the complement of $H+\bar{H} \cdot K$ nor the complement of $K+\bar{K} \cdot H$ cuts between any two points of $H \cdot K$ and, by Theorem 6 , the complement of $\bar{H} \cdot K+H \cdot \bar{K}$ does not.

This type of argument gives the following result.

THEOREM 11. The sum of two connected domains one of which is compact separates the plane provided their common part is not connected.

\section{REFERENCES}

1. S. Eilenberg, Transformations continues en circonférence et la topologie du plan, Fund. Math. vol. 26 (1936) pp. 61-112.

2. Z. Janiszewski, Sur les coupures $d u$ plan faites par les continus (in Polish), Prace matematyczno-fizyczne vol. 26 (1913) pp. 11-63.

3. F. B. Jones, Certain consequences of the Jordan Curve Theorem, Amer. J. Math. vol. 63 (1941) pp. 531-544.

4. B. Knaster and C. Kuratowski, Sur les continus non-bornés, Fund. Math. vol. 5 (1924) pp. 23-58.

5. - - Sur les ensembles connexes, Fund. Math. vol. 2 (1921) pp. 206-255.

6. C. Kuratowski and S. Straszewicz, Généralisation d'un théorème de Janiszewski, Fund. Math. vol. 12 (1928) pp. 152-157.

7. C. Kuratowski, Sur la séparation d'ensembles situés sur le plan, Fund. Math. vol. 12 (1928) pp. 214-239.

8. R. L. Moore, Foundations of point set theory, Amer. Math. Soc. Colloquium Publications, vol. 13, New York, 1932.

9. Anna M. Mullikin, Certain theorems relating to plane connected sets, Trans. Amer. Math. Soc. vol. 24 (1922) pp. 144-162.

10. S. Nikodym, Sur les coupures du plan faites par les ensembles connexes et les continus, Fund. Math. vol. 7 (1925) pp. 15-23. 
11. S. Straszewicz, Über die Zerschneidung der Ebene durch abgeschlossene Mengen, Fund. Math. vol. 7 (1925) pp. 159-187.

12. - Über eine Verallgemeinerung des Jordan'schen Kurvensatzes, Fund. Math. vol. 4 (1923) pp. 128-135.

The UnIversity OF TeXas

\section{ON ISOMETRIES OF SQUARE SETS}

PAUL J. KELLY

1. Introduction. It is not fully known under what conditions the isometry of two square, metric sets, say $E^{2}$ and $F^{2}$, implies the isometry of $E$ and $F$. Using the notion of order two self-isometries, this paper gives conditions sufficient to imply $E$ isometric to $F$ when $E^{2}$ and $F^{2}$ are finite and are metrized under any one of a fairly extensive class of functions. The basic ideas are first applied to non-square sets to yield a more general theorem which is then applied to the inverse square problem.

2. Definitions. A set is called metric if to every pair of its elements, $a$ and $b$, there corresponds a real, non-negative number, which is independent of the order of $a$ and $b$, zero if and only if $a$ equals $b$, and which satisfies the triangle law.

Two metric sets are isometric (written " $\equiv "$ ) if there is a one-to-one transformation of one set on the other in which the metric number associated with any pair is the same as that associated with the transformed pair.

A non-identity mapping of a set on itself, which is an isometry, and which leaves each element of the set invariant or else interchanges it with another, is called a self-isometry of order two. Any subset on which the self-isometry is the identity is said to be left pointwise invariant.

Theorem 1. Assume $A \equiv B$ under a mapping $T$, where $A$ and $B$ are finite metric sets. Let $A$ and $B$ have self-isometries of order two under mappings $R$ and $S$ respectively and let $A_{1}$ and $B_{1}$ denote respectively the maximum subsets left pointwise invariant. If $A_{1}$ has no self-isometry of order two, and has at least as many elements as $B_{1}$, then $A_{1} \equiv B_{1}$ and there

Presented to the Society, November 25, 1944, under the title Some properties of a certain interchange type of self-isometry; received by the editors September 23, 1944. 